\title{
Multi Criteria Adaptation Mechanisms in Homological Sensor Networks
}

\author{
Hui Zhang \\ School of Computer Science \\ and Informatics \\ University College Dublin \\ Dublin, Ireland \\ Email: hui.zhang@ucd.ie
}

\author{
Paddy Nixon \\ School of Computer Science \\ and Informatics \\ University College Dublin \\ Dublin, Ireland \\ Email: paddy.nixon@ucd.ie
}

\author{
Simon Dobson \\ School of Computer Science \\ and Informatics \\ University College Dublin \\ Dublin, Ireland \\ Email: simon.dobson@ucd.ie
}

\begin{abstract}
One fundamental feature of sensor network is unpredictable and this brings us a challenge of how to design an adaptation mechanism to let a sensor network adjust itself based on current "context" and pre-defined network criteria which include coverage density, fault tolerance, system life and so on.

The key idea of our work is, based on calculation of nodes coverage, we can let networks be adaptive to ongoing changing network situation. We use system working life as our criterion in this work to demonstrate the potential usage of our system.

An algebraic topology tool, homology, is used in our work to calculate sensor nodes coverage since the local properties of a sensor network, obtained by local interactions among nodes, can be captured by certain topological spaces. Also the global properties of the sensor network characteristics correspond to certain topological invariants of these spaces.

Unlike other approaches, our work does not need any node location or orientation information and it does not have any assumption about the node deployment control and domain geometry. The only thing that it needs to calculate nodes coverage is a peer to peer communication graph.
\end{abstract}

\section{INTRODUCTION}

With the fast development of micro-electro-mechanical systems (MEMS) technology, digital electronics and wireless communications, we can build low-cost, small size, low-power and multifunctional sensors which can communicate with each through wireless signal in short distance. These sensors with sensing, data processing and wireless communication abilities, based on collaborative effort, make building a largescale wireless sensor network possible and ensure a wide range of sensor network applications which include health, military, security and smart home [1], [2], [3], [4], [5]. We firmly believe that in future sensor networks will become an important part of our life.

Sensor networks is composed of a large number of sensor nodes which are densely deployed inside or close to the phenomenon. The location of the sensor nodes are not predetermined. Most case they are deployed randomly in the unaccessible terrain and are moving from time to time during the disaster relief operation. This also means that sensor node needs self-organizing and collaborative capability to work together in order to achieve its goal. Each sensor node monitors the environment and generates data to deliver to remote base station. The base station then analyzes and processes the data to decide whether there is an unusual event appeared in the monitored area. Considering the large number of deployed sensor nodes, the redundancy can be exploited to increase data accuracy and system reliability.

The power supply to sensor nodes in a network is from battery which can only last for a limited life time and make it impossible for a sensor node working for a very long time without recharging. Moreover most sensor network applications are deployed in hostile or remote areas, such as battlefield or forest which make it difficult to replace or recharge node batteries.

New nodes can join the network and some nodes can be damaged or out of power supply, so one fundamental feature of sensor network is unpredictable. The network is changing all the time. This brings us a new challenge to design an adaptive mechanism which allows a sensor network to configure itself based on some criteria according to current network situation. The criteria are user-defined such as nodes density, detection fault tolerance, nodes coverage, system working life and so on. All these criterion are based on calculation of network coverage, which describes how well a targeted area is monitored by a selection of sensor nodes, hence, given a group of sensor nodes, to calculate their coverage becomes the most important task in our work.

Our work is driven by the following factors. First, it is impossible to manually control the node in a network, so the sensor node has to be able to self-configurate. Second, each sensor node does not know its location, since to equip the sensor with GPS device will make sensor node consume much more power and this does not obey the idea of "small size and low-cost node".

In this work,inspired by the idea first presented by Robert Ghrist [6], we implement an algorithm to calculate sensor coverage. Algebraic topology tool, homological group, is used to calculate the network coverage without the knowledge of sensor nodes location and target area coordinate.

To demonstrate the usage of the system, we pick system life time as our criterion. Our goal is to let network maximize the system life and at the same time maintain the network full coverage. The key idea is that in a wireless sensor network all sensor nodes have the same sensing task which implies only 
part of sensor nodes are required to be active to carry out sensing task during the entire system life. Switching off some redundant nodes which does not reduce system performance and nodes coverage can prolong the system life time. With the network coverage information, different node configurations of minimum coverage can be calculated. Then an energy-efficient node scheduling can be obtained.

In the proposed approach, each sensor node only knows its neighbors, nodes that are within its radio communication range, by broadcasting its unique ID. Then sensor node forwards this communication information to base station. The base station node uses the proposed algorithm to compute network coverage and node configuration and finally informs those according nodes to work in group.

The rest of paper is structured as follows. The reviews of related work in the literature is presented in section 2 . In section 3 we give a brief introduction to simplicial complexes, homology groups. Section 4 describes details of the proposed approach and algorithm implementation. Finally simulation results and evaluation are given in Section 5.

\section{RELATED WORK}

In order to compute the sensor network coverage different approaches have been presented. Generally, they can be divided into three groups.

The first approach is called computational geometry method [7], [8], [9]. One feature of this approach is that the precise geometry of the domain and exact locations of the nodes must be known.

The second approach is probabilistic method [10], [11], [12], [13]. They assume a randomly and uniformly distribute sensor nodes in a domain with a fixed geometry and prove expected area coverage. The main drawback of this method is the need for strict assumptions about the exact shape of the domain as well as the need for a uniform distribution of nodes.

The third approach is called algebraic topology method which uses network topological spaces and their topological invariants. The idea behind this is that the local properties of a sensor network, obtained by local interactions among nodes, can be captured by certain topological spaces. Also the global properties of the sensor network characteristics correspond to certain topological invariants of these spaces. Some attempts from theocratic concepts of computing sensor network coverage have been made in [14], [15], [16]

Our proposed solution uses algebraic topology tool to calculate minimum network coverage without any information about node location, orientation and domain geometry. Based on the calculation of the network coverage, our algorithm is able to find several mutually exclusive node sets which can be scheduled to work in order. Then the overall network life time can be prolonged.

\section{Simplicial Complex ANd Homology}

The mathematical tools being used in our work might not be known by researchers in wireless sensor networks. A brief introduction is presented below. Further readings of various

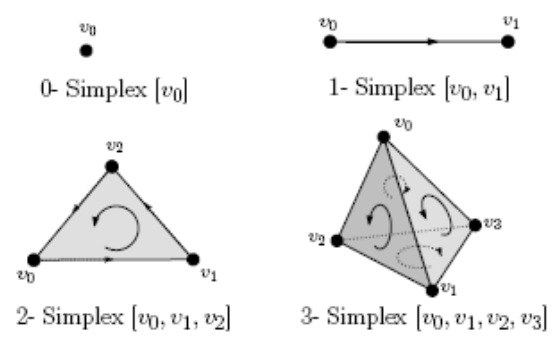

Fig. 1. Simplices example

degree of depth can be found at [17], [18], [19]. In [20] the context of related applications and computations are described.

Simplicial Complex: The topological objects used in our work are called simplicial complexes. Given a set of points $V$, a $k$-simplex is an unordered subset $\left\{v_{0}, v_{1}, \cdots, v_{k}\right\}$ where $v_{i} \in V$ and $v_{i} \neq v_{j}$ for all $i \neq j$. The faces of a ksimplex consist of iiiiiii .mine simplices which s closed with respect inclusion of faces. A good example is a ======= all $(k-1)$-simplices of the form $v_{0}, \ldots, v_{i-1}, v_{i+1}, \ldots, v_{k}$ for $0 \leq i \leq k$. A simplicial complex is a collection of simplices which $\mathrm{s}$ closed with respect inclusion of faces. A good

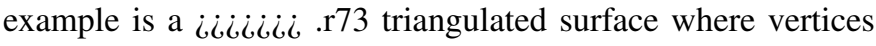
of the triangulation correspond to $V$, edges correspond to 1simplices, and face correspond to 2-simplices. The orderings of vertices correspond to an orientation.

Simplicial Homology: Homology is an algebraic procedure of counting 'holes' of various types. Let $X$ denote a simplical complex. The homology of $X$, denoted $H_{*}(X)$, is a sequence of vector spaces $H_{k}(X): k=0,1,2,3 \cdots$, where $H_{k}(X)$ is called the $k$-dimensional homology of $X$. The dimension of $H_{k}(X)$, called the $k^{t h}$ Betti number of $X$, is a coarse measurement of the number of different holes in the space $X$ than can be sensed by using subcomplexes of dimension $k$.

For example, the dimension of $H_{0}(X)$ is equal to the number of path-connected components of $X$ and the simplest basis for $H_{1}(X)$ consists of loops in $X$, each of which surrounds a different 'hole' in $X$.

Let $X$ denote a simplical complex. Define for each $k \geq 0$, the vector space $C_{k}(X)$ to be the vector space whose basis is the set of oriented $k$-simplices of $X$; that is, a $k$-simplex $\left\{v_{0}, \cdots, v_{k}\right\}$ together with an order type denoted $\left[v_{0}, \cdots, v_{k}\right]$ where a change in orientation corresponds to a change in the sign of the coefficient:

$\left[v_{0}, \cdots, v_{j}, \cdots, v_{i}, \cdots, v_{k}\right]=-\left[v_{0}, \cdots, v_{i}, \cdots, v_{j}, \cdots, v_{k}\right]$.

For $k$ larger than the dimension of $X, C_{k}(X)=0$. The boundary map is defined to be the linear transformations $\partial: C_{k} \rightarrow C_{k-1}$ which acts on basis elements $\left[v_{0}, \cdots, v_{k}\right]$ via

$$
\partial\left[v_{0}, \cdots, v_{k}\right]:=\sum_{i=0}^{k}(-1)^{i}\left[v_{0}, \cdots, v_{i-1}, v_{i+1}, \cdots, v_{k}\right]
$$

, as illustrated in Fig 2. 

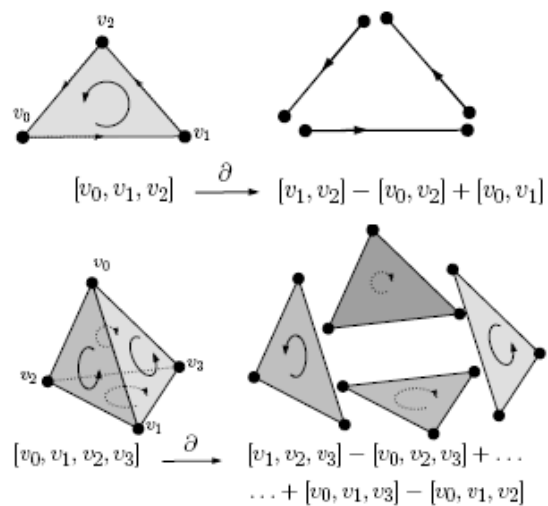

Fig. 2. The boundary operator on a 2-simplex and 3-simplex

Given the definition of boundary map, we can define a chain complex: a sequence of vector spaces and linear transformations

$$
\cdots \stackrel{\partial}{\longrightarrow} C_{k+1} \stackrel{\partial}{\longrightarrow} C_{k} \stackrel{\partial}{\longrightarrow} C_{k-1} \cdots \stackrel{\partial}{\longrightarrow} C_{1} \stackrel{\partial}{\longrightarrow} C_{0}
$$

Consider the following two subspaces of $C_{k}$ : the cycles (those subcomplexes without boundary) and the boundaries (those subcomplexes which are themselves boundaries).

$$
\begin{gathered}
k-\text { cycles }: Z_{k}(X)=\operatorname{ker}\left(\partial: C_{k} \rightarrow C_{k-1}\right) \\
k \text { - boundaries }: B_{k}(X)=\operatorname{im}\left(\partial: C_{k+1} \rightarrow C_{k}\right)
\end{gathered}
$$

It can be easily demonstrated that $\partial \circ \partial=0$; which means the boundary of a chain has empty boundary. It follows that $B_{k}$ is a subspace of $Z_{k}$. We say that two cycles $\xi$ and $\eta$ in $Z_{k}(X)$ are homologous if their difference is a boundary:

$$
[\xi]=[\eta] \leftrightarrow \xi-\eta \in B_{k}(X)
$$

The $k$-dimensional homology of $\mathrm{X}$, denoted $H_{k}(X)$ is the quotient vector space,

$$
H_{k}(X)=\frac{Z_{k}(X)}{B_{k}(X)}
$$

\section{Coverage Calculation and Node Scheduling}

In this section, the practical issues in understanding and realizing homologoy groups in real networks are discussed.

\section{A. Communication Graphs and Rips Complexes}

We first explain the relationship between simplicial complexes and sensor network coverage. As presented in [14], [15], [16], the sensing and communication properties of sensor network can be captured by simplicial complexes and their homological groups. Considering in a wireless sensor network, each identical node has same communication range $r$ and they form a communication graph. In this graph, each vertex stands for a sensor node and an edge between two vertices means that the two nodes are within the communication range $r$ of each other. We now build the rips complex which is generated from communication graph.
Definition IV-A.1. Given a set of points $v_{1}, \cdots, v_{n}$ in $\mathbb{R}^{d}$ in Euclidean d-space and a fixed radius $r>0$, the rips complex $\mathcal{R}$, is the simplicial complex whose $k$-simplices correspond to the unordered $(k+1)$-tuples of points which are pairwise within a distance $r$ of each other.

The 0-simplices are the nodes in the communication graph and 1-simplices are all the edge in the graph. Thus, the 2simplices are the triangle in the graph which has three nodes and each node is within communication range of the other two. The dimension of zero homology group $H_{0}(\mathcal{R})$ counts the number of connected components of the network. For example, the communication graph is connected if and only if $H_{0}(\mathcal{R})$ has dimension of 1 . The homology group $H_{1}(\mathcal{R})$ counts the number of the network holes in the communication graph. The hole appears when the part of graph can not be triangulated by any 2-simplices. As shown in [14], [15], [16], these homology groups are related to certain coverage properties of a sensor network.

In [14], author gives and proves a theorem listed below which can be used to calculate the network coverage based on some assumptions. The assumptions they have are: First, the identical sensor node has radially symmetric covering domains $r_{c}$ which is at least larger than $r_{b} / \sqrt{3}$ and second, the communication graph is connected. Third there are fence nodes which are fixed along the edge of targeted area.

Theorem IV-A.2. For a set of nodes $\mathcal{X}$ in a domain $\mathcal{D} \subset \mathbb{R}^{2}$ satisfying those assumptions, the sensors cover whole domain $\mathcal{D}$ if there exits $[\alpha] \in H_{2}(\mathcal{R}, \mathcal{F})$ such that $\partial \alpha \neq 0 . \mathcal{F}$ is a particular cycle traversing the fence nodes.

A detailed prove about this theorem is provided in their work [14].

\section{B. Coverage Calculation}

Inspired by the idea of R. Ghrist [14], we develop the practical algorithm to calculate the coverage and simulate it in ns-2.

As discussed above, in order to calculate the $H_{0}(\mathcal{R})$ and $H_{1}(\mathcal{R})$ groups, we have to first get the simplicial complex from communication graph: each node first broadcasts its unique ID to all its neighbors who can detect this message. Once each neighbor gets this request it will check its membership of different dimensional simplices.

For 0-simplex and 1-simplex membership calculation, it is very straightforward. Node just adds its ID to 0-simplices list and add its own ID plus package sender as a new 1simplex. For 2-simplices list, the node has to calculate and search through the simplices it already has to check if there is a combination which can form a triangle.Here is the algorithm:

The whole process is shown in the Fig 3.

Once every sensor node gets its membership of 0 to 2 dimension simplices, it then forwards these information to base station where the homological calculation takes place.

The calculation of homology is by no means of novel and for detailed description of related algorithms please refer to 


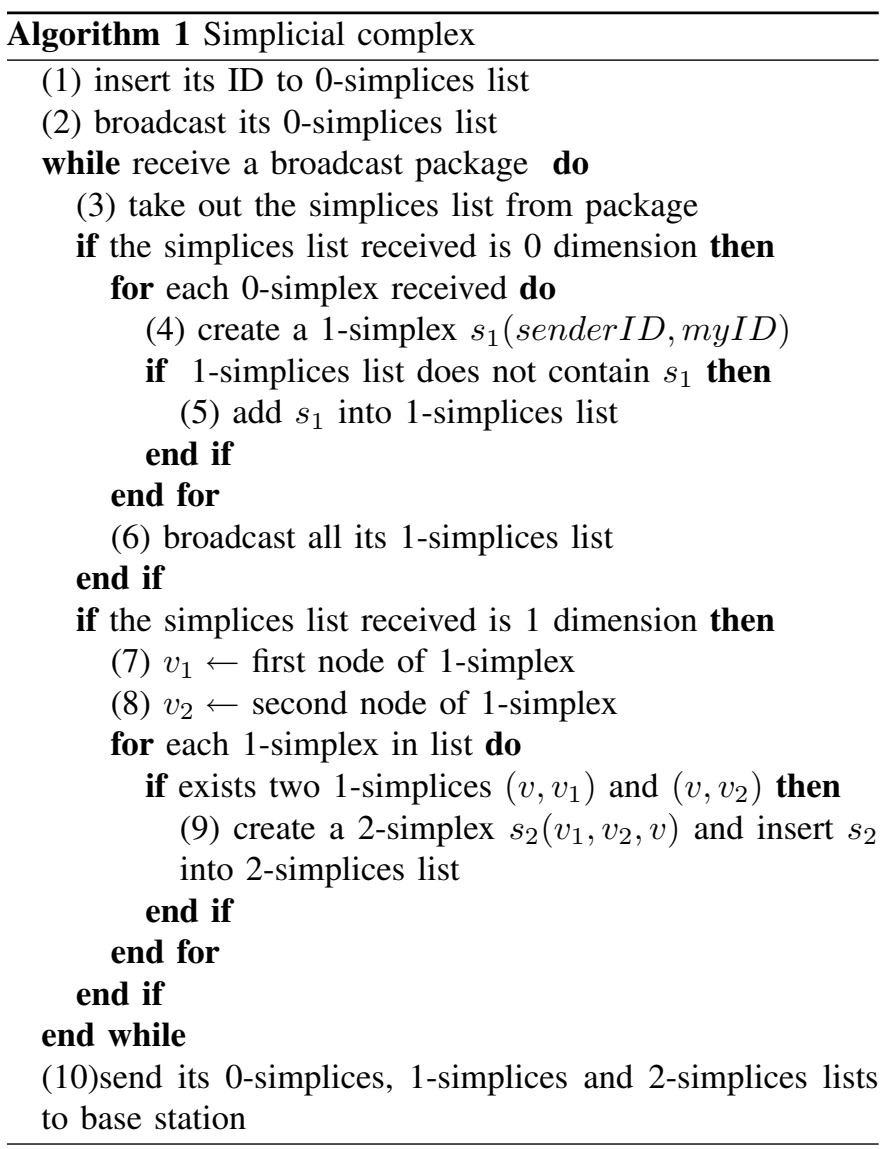

[20]. First, the base station node has to calculate $H_{0}(\mathcal{R})$ and $H_{1}(\mathcal{R})$ to make sure the betti numbers are 1 and 0 respectively, which means currently all sensor nodes provide a connected communication graph and full coverage of the area.

We assume that if the node density and redundant rate are high enough there will always be at least two disjoined sets of nodes and each of which can achieve a full coverage of the area.

The algorithm first computes the minimum number of working nodes which theoretically can cover the whole area with uniform separated distance. And it randomly picks this number of nodes and tries to calculate the coverage till a full coverage is found. It calculates an overall attempt times based on the probability to find a full coverage. If after all the attempts, a full coverage is still not found, the algorithm increases the number of working nodes by one and re-calculates the attempt time. Once the first set of full coverage nodes is found, it removes these nodes from the total node set and then find the next full coverage set from the rest nodes until there is no more set can be found.

When these disjoined sets are found, the base station then schedules them to work in order.

Algorithm 2 shows the details.

\section{Simulation And Evaluation}

We simulate our algorithm in ns-2 [21]. With total number of nodes from 20 to 50 plus 12 fence nodes. The targeted area

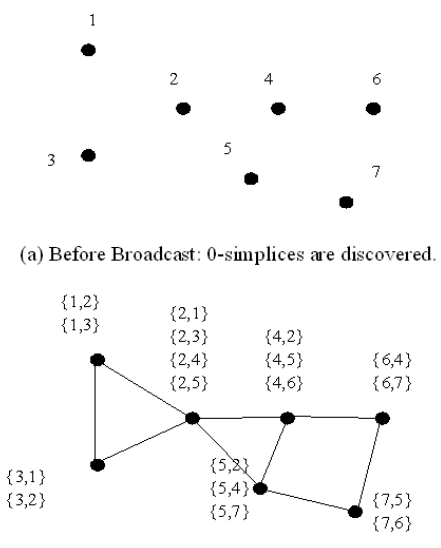

(b) Before Broadcast: 1-simplices are discovered.

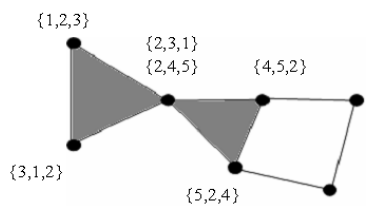

(c) Before Broadcast: 2 -simplices are discovered

Fig. 3. The 3-round broadcasting process to calculate simplicial complex

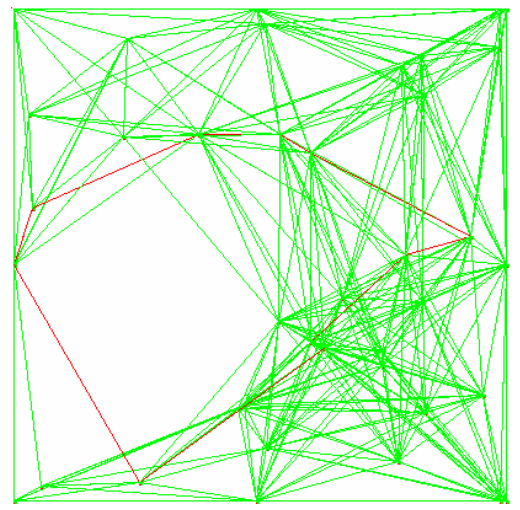

Fig. 4. Sensor network has one coverage hole

has a size of 750 by 750 and the node communication range is 250. Simulation results with visualizations are depicted.

If the node density is not high or the nodes are not distributed uniformly enough, there will be a coverage hole. In this situation, base station will be aware of this since the betti number of $H_{1}(\mathcal{R})$ is not 0 . As depicted in Fig 4, there is one hole in this example, circulated by the red color cycle. Actually the hole is caused by 4 nodes down the left corner, if we keep reducing the length of this red cycle (by finding the minimum size of $H_{1}(\mathcal{R})$ generator), we will finally be able to locate this hole. But if there is more than one hole, it will be 

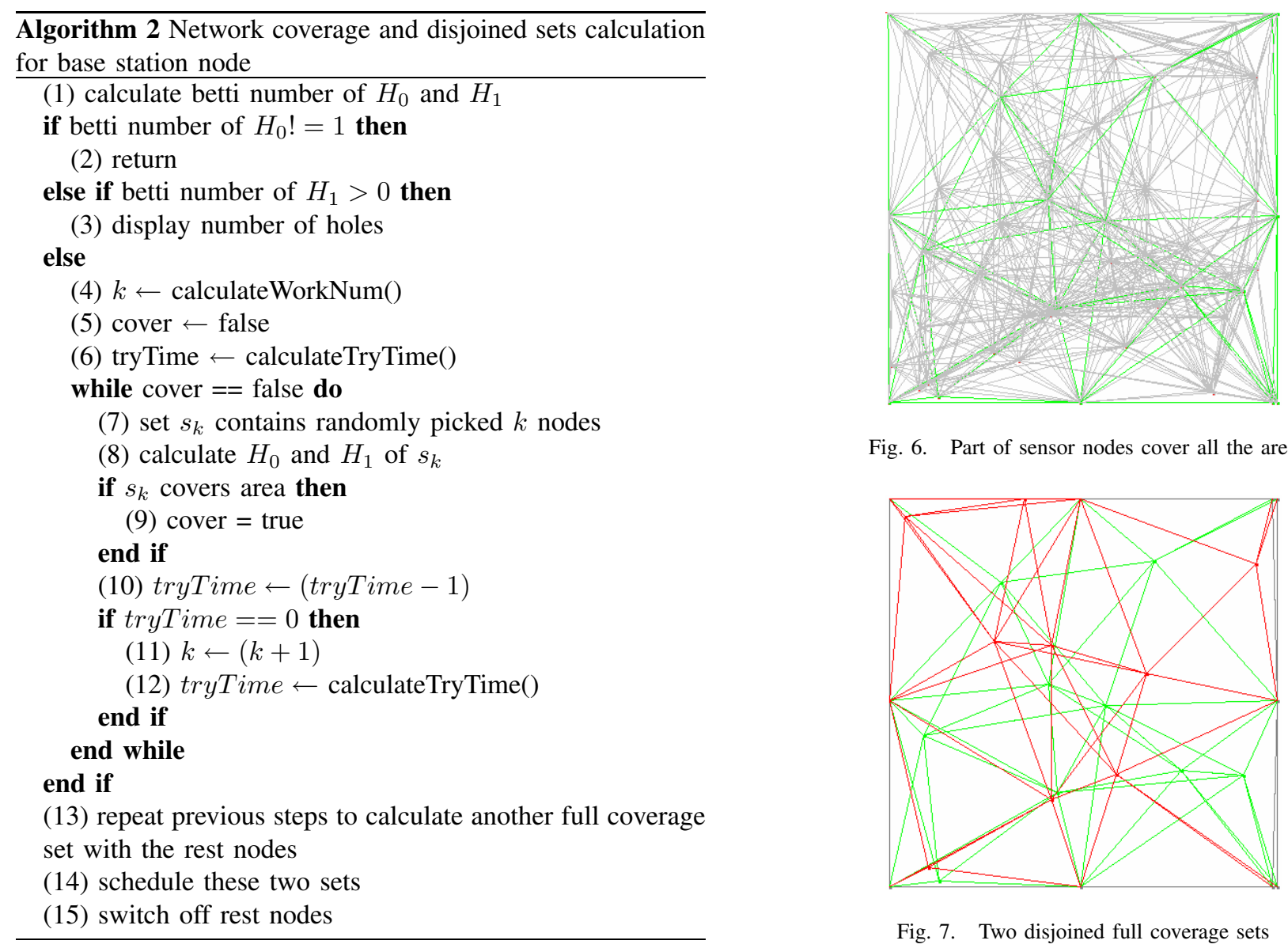

Fig. 6. Part of sensor nodes cover all the area

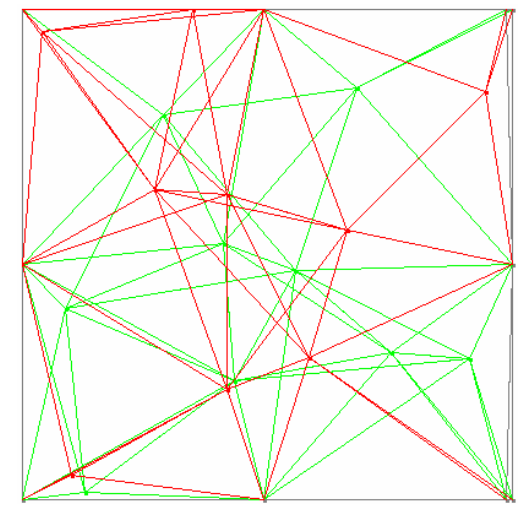

Fig. 7. Two disjoined full coverage sets
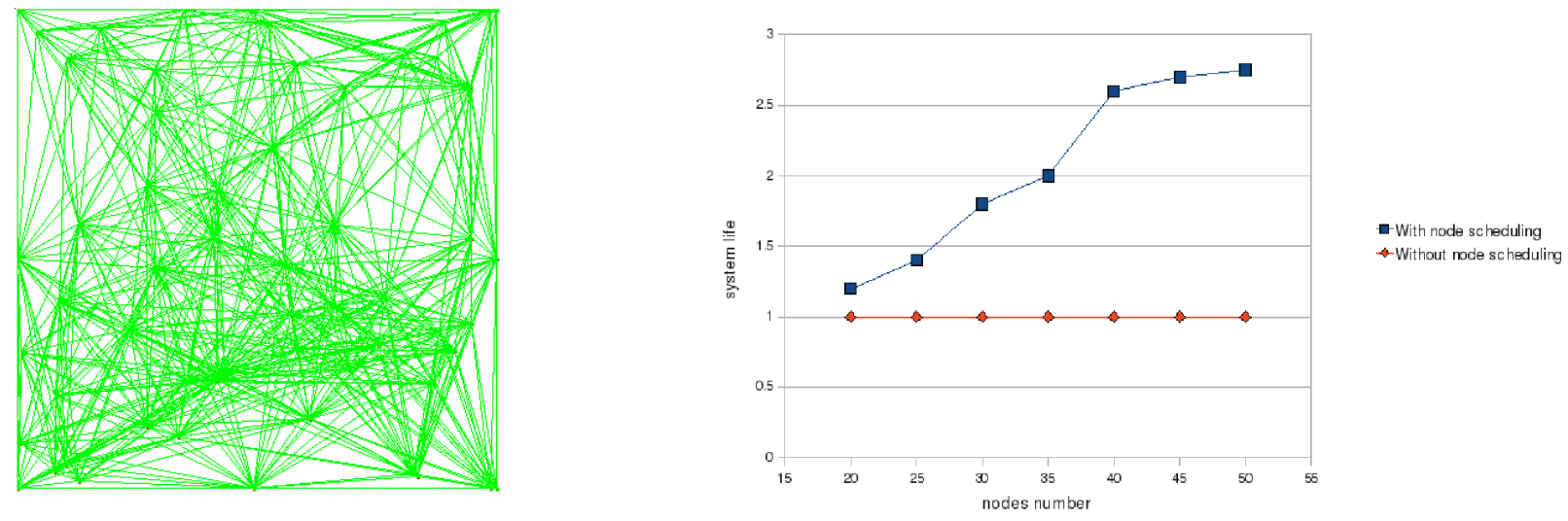

Fig. 5. Sensor network has a full coverage

Fig. 8. Increased system life when different number of nodes are deployed.

difficult to locate exact positions for both of them.

If the node density is high enough, there will be more likely that the nodes can cover all the area without any coverage hole. A full coverage example is shown in Fig 5.

By randomly picking a set of nodes with certain size from the current communication graph, we can calculate if the set reserves the whole area coverage property or not. In Fig 6, it shows a set of 9 nodes which can achieve a full coverage. And rest of nodes, colored in dark gray, can be switched off to save power.

Finally from the rest of nodes without the nodes of set which

already covers the area, algorithm picks another full coverage set of nodes which is totally disjoined from the first one. Two sets are drawn out in Fig 7. The green set is the first one and the red is the second set. Each of them covers the whole area.

The system life-time can be prolonged by scheduling these two sets to work in turn and switch off the rest redundant nodes. Based on different sizes of deployed nodes, the algorithm performance varies. As shown in Fig 8. We can see that when the total nodes number is larger than a threshold value (40, in this case) the system life increases will be much slower. 


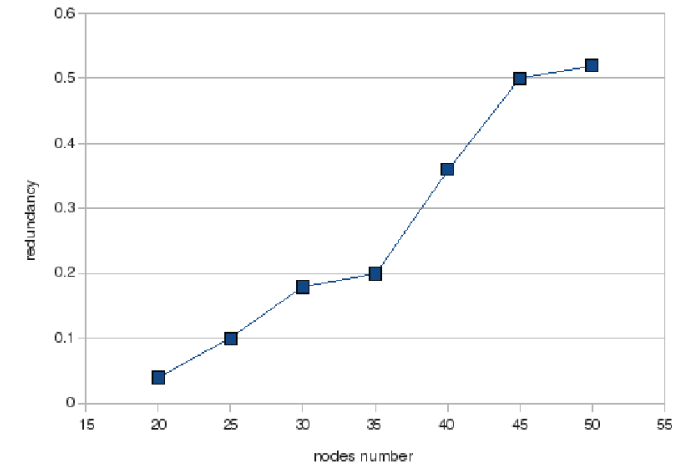

Fig. 9. Increased average redundancy (per working nodes set) when different number of nodes are deployed.

This is because we are randomly picking nodes to calculate coverage and when the total number is large the chance to find most fitted sets (with as few nodes as possible) is smaller and the redundancy of each working set is higher as shown in the Fig 9.

\section{Summary AND CONCLUSIONS}

In this work, we implemented an algorithm to calculate sensor coverage inspired by the idea first presented by Robert Ghrist [6]. Algebraic topology tool - homological group is used to calculate the network coverage without the knowledge of sensor nodes location and target area coordinate. With the network coverage information, a wireless sensor network then can adjust itself based on current network topology. We demonstrate that the algebraic topology tool could be used in a practical design of wireless sensor network and has great potential for various sensor network applications as well. However, the fundamental homological coverage calculation carried out by the base station node is still centralized. A decentralized algorithm is required to support large-scale sensor networks.

\section{REFERENCES}

[1] I.A. Essa. Ubiquitous sensing for sart and aware environments. IEEE Personal Communication, pages 47-49, October 2000.

[2] Herve V. Rialle G. Virone E. Mercier G. Morey A. Moro T. Porcheron N.Noury, T. Monitoring behavior in home using a smart fall sensor. IEEE-EMBS Special Topic Conference on Microtechnologies in Medicine and Biology, pages 607-610, October 2000.

[3] J.L. da Silv Jr. D. Patel S. Roundy J.M. Rabaey, M.J. Ammer. Picoradio supports ad hoc ultra-low power wireless networking. IEEE Computer Magazine, pages 42-48, 2000.

[4] B. Sibbald. Use computerized systems to cut adverse drug events: report. CMAJ: Canadian Medical Association Journal, 2001.

[5] http://www.alertsystems.org.

[6] R. Ghrist and A. Muhammad. Coverage and hole detection in sensor networks via homology. Proc. IPSN, 2005.

[7] M. Potkonja S. Meguerdichian, F. Koushanfar and M. B. Srivastava. Coverage problems in wireless ad-hoc sensor netowrks. IEEE INFOCOM, pages 1380-1387, 2001.

[8] D. Tian and N. D. Geogranas. A coverage-preserving node scheduling scheme for large wireless sensor networks. ACM Int'l Workshop on Wireless Sensor Networks and Applications (WSNA), 2002.

[9] H. Zhang and J. Hou. Maintaining coverage and connectivity in large sensor networks. invited paper in International Workshop on Theoretical and Algorithmic Aspects of Sensor, Ad hoc Wireless and Peer-toPeer Networks, Florida, Feb 2004
[10] H. Koskien. On the coverage of a random sensor network in a bounded domain. 16th ITC Specialist seminar, pages 11-18, 2004.

[11] B. Liu and D. Towsley. A study of the coverage of large-scale sensor networks. IEEE Int'l Conf on Mobile Ad-hoc and Sensor Systems, 2004.

[12] F. Xue and P. R. Kumar. The number of neighbors needed for connectivity of wireless networks. Wireless Netowrks, (2):169-181, March 2004.

[13] C. Hsin and M. Liu. Network coverage using low duty-cycled sensors: Random and coordinated sleep algorithms. Information Processing in Sensor Networks (IPSN), April 2004.

[14] V. de Silva and R. Ghrist. Homological sensor networks. Notices American Mathematical Society, pages 10-17, 2007.

[15] V. de Silva and R. Ghrist. Coordinate-free coverage in sensor networks with controlled boundaries via homology. Intl. J. Robotics Research, 25(12):1205-1222, 2006.

[16] V. de Silva and R. Ghrist. Coverage in sensor networks via persistent homology. Alg. Geom. Topology, 7:339-358, 2007.

[17] A. Hatcher. Algebraic topology. Cambridge University Press, 2002.

[18] J. Munkres. Elements of algebraic topology. Addison Wesley, 1993.

[19] M. A. Armstrong. Basic topology. Springer-Verlag, 1997.

[20] K. Mischaikow T. Kaczynski and M. Mrozek. Computational homology. Applied Mathematical Sciences 157, Springer-Verlag, 2004.

[21] http://www.isi.edu/nsnam/ns/. 